\title{
"Wood already touched by fire is not hard to set alight" \\ Comment on "Constraints to applying systems thinking concepts in health systems: A regional perspective from surveying stakeholders in Eastern Mediterranean countries"
}

\author{
Irene Akua Agyepong ${ }^{1,2^{*}}$
}

\section{Abstract}

A major constraint to the application of any form of knowledge and principles is the awareness, understanding and acceptance of the knowledge and principles. Systems Thinking (ST) is a way of understanding and thinking about the nature of health systems and how to make and implement decisions within health systems to maximize desired and minimize undesired effects. A major constraint to applying ST within health systems in Low- and Middle-Income Countries (LMICs) would appear to be an awareness and understanding of ST and how to apply it. This is a fundamental constraint and in the increasing desire to enable the application of ST concepts in health systems in LMIC and understand and evaluate the effects; an essential first step is going to be enabling of a wide spread as well as deeper understanding of ST and how to apply this understanding.

Keywords: Health Systems, Systems Thinking (ST), Decision-making, Low- and Middle-Income Countries (LMIC)

Copyright: ๑ 2015 by Kerman University of Medical Sciences

Citation: Agyepong IA. "Wood already touched by fire is not hard to set alight": Comment on "Constraints to applying systems thinking concepts in health systems: A regional perspective from surveying stakeholders in Eastern Mediterranean countries". Int J Health Policy Manag 2015; 4: 191-193. doi: 10.15171/ijhpm.2015.34

\section{Article History:}

Received: 28 January 2015 Accepted: 13 February 2015 ePublished: 15 February 2015

\section{Introduction}

At the heart of Systems Thinking (ST) is the recognition of a whole made up of separate and yet linked and interdependent parts that interact with each other to make the whole function well. Occurrences and outcomes can only be properly understood by appreciating this interconnectedness $(1,2)$. Health systems moreover are complex and adaptive rather than mechanical systems. They comprise structures and institutions; but also processes and people. They are moreover integral parts of the socio-economic, political, demographic, geographic, etc. contexts or environments, within which they exist. These contexts are themselves complex and adaptive. To try to attain the optimal functioning of part of a system, while ignoring the other parts and the interconnectedness may lead to short-term gains. In the medium to long-term it often results in some form of weakness and difficulties in sustaining the initial gains. The components of a system work best when the whole is also working well. The experience of Ghana in the implementation of the Additional Duty Hours Allowance (ADHA) policy is an example of this kind of short-term success, followed by medium and long-term difficulties (3).

"Systems thinking" as the name implies is a way of understanding and conceptualizing the world, thinking and reasoning. To be able to apply this way of understanding and conceptualizing the world to decision-making in health or any other sector for that matter; requires the devising and application of tools and approaches. However the tools and approaches are not themselves ST. Rather, a clear understanding of ST concepts makes it possible to adapt already existing tools and approaches and develop new ones appropriately in context.
Growth in publications that use the terms: "Systems thinking", "complex adaptive systems", and or "systems science" have increased exponentially (4) in recent years; as has interest in the application of ST to support decisionmaking and implementation related to promoting, improving and maintaining health. The growing literature is however dominated by papers with corresponding authors from HighIncome Countries (HIC) in North America and Europe. Lowand Middle-Income Countries (LMIC) remain relatively under represented (4). The Alliance for Health Policy and Systems Research flagship report, "Systems Thinking for Health Systems Strengthening" (5) and subsequent efforts to generate interest, promote and raise awareness of the potential of ST in health systems in LMIC has in recent years contributed to efforts to bridge the gap between research publications on ST between HIC and LMIC. The paper by El-Jardali et al. (6) is one of the few but growing papers led by authors from LMIC on ST that has resulted from this effort.

\section{Objectives}

This commentary reflects further on some of the issues raised in this paper related to the application of ST in health system decision-making as well as the implementation and monitoring of decisions in LMIC, and the constraints and enablers. It is especially concerned with what it will take to make ST an integral part of decision-making and implementation processes in health systems.

Insights from Low- and Middle-Income Countries (LMIC) in the Eastern Mediterranean Region (EMR)

El-Jardali et al. (6) paper provides insights into how ST concepts have been received and used by health systems 
stakeholders such as senior officials in the Ministry of Health $(\mathrm{MoH})$, researchers, civil society groups and professional associations from 10 countries in the Eastern Mediterranean Region (EMR). It also provides insights based on the opinions of these stakeholders on the key enablers and barriers to the wide use of ST concepts in research and decision-making and possible strategies to overcome these constraints.

The paper is made doubly interesting by its focus on ST in LMIC, a neglected area of work; and by its focus on 10 LMIC countries in the EMR. In the general relative dearth of published work in ST from LMIC, countries in the EMR stands out as meriting increased attention and support. A simple January 2015 Medline search showed that of the 1,166 publications that mentioned the EMR, only 13 papers used the term Systems Science. These papers but did not necessarily have ST as their core focus. No paper used the term "Complex Adaptive Systems" and only the paper by ElJardali and colleagues used the term ST.

The methodology involved a purposive sampling of respondents in each country. The response rate ranged from $17 \%-100 \%$ per country with an average of $41 \%$. It was not the stated intent of the study; and its results cannot be assumed to be statistically generalizable across the sub-region. The qualitative insights it provides are nevertheless important. Such insights are essential prerequisites to developing theories and frameworks that can inform further work, including more generalizable research designs.

The study highlights several constraints to as well as enablers of the application of ST within the health systems of the ten countries studied. Though on a positive note, most respondents had some idea of ST before the study; the depth of knowledge was limited, and there were misconceptions. This limitation appeared to cut across the researchers as well as the decision-makers in the sample. Thus potential users of ST to support decision-making and implementation processes within the health system; as well as producers of knowledge to inform the processes were challenged in the depth of their knowledge of ST as well as in the holding of misconceptions. Under these circumstances; it is not surprising that ElJardali et al. (6) found that despite strong recognition of the usefulness of ST there was very limited experience with applying ST; and reactive and fragmented approaches to decision-making. Limited experience with applying ST alone does not explain reactive and fragmented approaches to decision-making within health systems. Classical theories of decision-making $(7,8)$ suggest that it is almost impossible to attain the conditions needed for perfectly rational and comprehensive decision-making that explores, evaluates all options, and perfectly takes into account intended and unintended consequences. It is not possible for decisionmakers to identify and evaluate all possible outcomes with precision, or to know all possibilities. The complex and adaptive nature of health systems means even with the best of efforts, there is always room for the "surprising and unexpected". Instead of engaging in the often near impossible task of finding the perfect or optimal solution, decisionmakers may select a reasonably close alternative that is more immediately and practically available (9).

Given the focus of ST, it can be theorized that understanding of and experience with ST would be expected to minimize reactive and fragmented approaches to decision-making - even if it cannot totally eliminate them. Unfortunately there is no research from LMIC - whether observational or experimental, proving or disproving this theory. It remains an urgent area for future research. My observation suggests that through experiential learning; experienced decisionmakers, from having to engage within systems and make things work over a long period of time, are aware of some of the principles of ST. They may not necessarily call it ST. However this observation needs to be more empirically validated. And regardless of the validity or otherwise of this observation; in my opinion, strengthening the indepth theoretical understanding of what ST is will reinforce experiential learning.

Conclusions: Enabling more widespread application of Systems Thinking (ST) in health in Low- and MiddleIncome Countries (LMIC)

To be able to apply ST to decision-making and implementation processes in health systems requires the adaptation of already existing tools and approaches. It also requires the devising and application of new ones. And to adapt or develop these tools requires a clear understanding of ST concepts. A limited understanding of ST, is therefore a major barrier to any efforts to enable its wide scale application to decision-making and implementation processes within health systems. Conversely a strong understanding and appreciation of what ST is a major first step to enable the wide spread application of ST within health systems.

A proverb of the Akan people of Ghana states: "wood touched by fire is not hard to set alight" (10). The implication is that with the right preparatory work and conditions, a difficult task can become much easier. Among the many barriers and enablers identified in the El-Jardali et al. study, perhaps the important first barrier to break down or conversely enabler to activate is achieving wide spread understanding of ST and how to apply it. Accompanying this, must be deepening experience in application. Then it will become possible to move to the next stage of evaluating and gaining further insights into the questions around under what conditions the application of ST works, for whom, why and what effects it produces.

\section{Ethical issues}

Not applicable.

Competing interests

Author declares that she has no competing interests.

Author's contribution

IAA is the single author of the manuscript.

\section{References}

1. Paina L, Peters DH. Understanding pathways for scaling up health services through the lens of complex adaptive systems. Health Policy Plan 2012; 27: 365-73.

2. Senge P. The Fifth Discipline. The Art and Practice of the Learning Organization. New York: Crown Publishing Group; 1990.

3. Agyepong IA, Kodua A, Adjei S, Adam T. When 'solutions of yesterday become problems of today': crisis-ridden decision making in a complex adaptive system (CAS)—-the Additional 
Duty Hours Allowance in Ghana. Health Policy Plan 2012; 27 Suppl 4: iv20-31. doi: 10.1093/heapol/czs083

4. Adam T. Editorial Advancing the application of systems thinking in health. Health Res Policy Syst 2014; 12: 50. doi: 10.1186/14784505-12-50

5. Alliance for Health Policy and Systems Research, World Health Organization. Systems Thinking for Health Systems Strengthening. Geneva, Switzerland: WHO: 2009

6. El-Jardali F, Adam T, Ataya N, Jamal D, Jaafer M. Constraints to applying systems thinking concepts in health systems: A regional perspective from surveying stakeholders in Eastern
Mediterranean countries. Int J Health Policy Manag 2014; 3: 399-407. doi: 10.15171/ijhpm.2014.124

7. Yehezkel Dror. Public Policy-making re-examined. New York: Leonard Hil Books; 1968

8. Charles E. Lindblom and David Braybrooke: A Strategy of Decision. New York: The Free Press; 1970

9. Herbert A. Simon: Administrative Behavior: As study of Decision making processes in Administrative Organization. 3rd edition. New York: The Free Press; 1976.

10. Appiah P. Appiah KA, Agyeman-Duah I. Bu me Be. Proverbs of the Akans. Oxfordshire, UK: Ayebia Clarke Ltd; 2007. 